\title{
Entity Annotation based on Inverse Index Operations
}

\author{
Ganesh Ramakrishnan, Sreeram Balakrishnan, Sachindra Joshi \\ IBM India Research Labs \\ IIT Delhi, Hauz Khas, \\ New Delhi, India \\ \{ganramkr, sreevb, jsachind\}@in.ibm.com
}

\begin{abstract}
Entity annotation involves attaching a label such as 'name' or 'organization' to a sequence of tokens in a document. All the current rule-based and machine learningbased approaches for this task operate at the document level. We present a new and generic approach to entity annotation which uses the inverse index typically created for rapid key-word based searching of a document collection. We define a set of operations on the inverse index that allows us to create annotations defined by cascading regular expressions. The entity annotations for an entire document corpus can be created purely of the index with no need to access the original documents. Experiments on two publicly available data sets show very significant performance improvements over the documentbased annotators.
\end{abstract}

\section{Introduction}

Entity Annotation associates a well-defined label such as 'person name', 'organization', 'place', etc., with a sequence of tokens in unstructured text. The dominant paradigm for annotating a document collection is to annotate each document separately. The computational complexity of annotating the collection in this paradigm, depends linearly on the number of documents and the cost of annotating each document. More precisely, it depends on the total number of tokens in the document collection. It is not uncommon to have millions of documents in a collection. Using this paradigm, it can take hours or days to annotate such big collections even with highly parallel server farms. Another drawback of this paradigm is that the entire document collection needs to be reprocessed whenever new annotations are required.
In this paper, we propose an alternative paradigm for entity annotation. We build an index for the tokens in the document collection first. Using a set of operators on the index, we can generate new index entries for sequences of tokens that match any given regular expression. Since a large class of annotators (e.g., GATE (Cunningham et al., 2002)) can be built using cascading regular expressions, this approach allows us to support annotation of the document collection purely from the index.

We show both theoretically and experimentally that this approach can lead to substantial reductions in computational complexity, since the order of computation is dependent on the size of the indexes and not the number of tokens in the document collection. In most cases, the index sizes used for computing the annotations will be a small fraction of the total number of tokens.

In (Cho and Rajagopalan, 2002) the authors develop a method for speeding up the evaluation of a regular expression ' $R$ ' on a large text corpus by use of an optimally constructed multi-gram index to filter documents that will match ' $R$ '. Unfortunately, their method requires access to the document collection for the final match of ' $R$ ' to the filtered document set, which can be very time consuming. The other bodies of related prior work concern indexing annotated data (Cooper et al., 2001; Li and Moon, 2001) and methods for document level annotation (Agichtein and Gravano, 2000; McCallum et al., 2000). The work on indexing annotated data is not directly relevant, since our method creates the index to the annotations directly as part of the algorithm for computing the annotation. (Eikvil, 1999) has a good survey of existing document level IE methods. The relevance to our work is that only a certain class of annotators can be implemented using our method: namely anything that can be implemented using cascading weighted regular expressions. Fortu- 
nately, this is still powerful enough to enable a large class of highly effective entity annotators.

The rest of the paper is organized as follows. In Section 2, we present an overview of the proposed approach for entity annotation. In Section 3, we construct an algorithm for implementing a deterministic finite automaton (DFA) using an inverse index of a document collection. We also compare the complexity of this approach against the direct approach of running the DFA over the document collection, and show that under typical conditions, the index-based approach will be an order of magnitude faster. In Section 4, we develop an alternative algorithm which is based on translating the original regular expression directly into an ordered AND/OR graph with an associated set of index level operators. This has the advantage of operating directly on the much more compact regular expressions instead of the equivalent DFA (which can become very large as a result of the NFA to DFA conversion and epsilon removal steps). We provide details of our experiments on two publicly available data sets in Section 5. Finally we present our conclusions in Section 6.

\section{Overview}

Figure 1 shows the process for entity annotation presented in the paper. A given document collection $\mathcal{D}$ is tokenized and segmented into sentences. The tokens are stored in an inverse index $I$. The inverse index $I$ has an ordered list $\mathcal{U}$ of the unique tokens $u_{1}, u_{2}, . . u_{W}$ that occur in the collection, where $W$ is the number of tokens in $I$. Additionally, for each unique token $u_{i}, I$ has a postings list $L\left(u_{i}\right)=<l_{1}, l_{2}, \ldots l_{c n t\left(u_{i}\right)}>$ of locations in $\mathcal{D}$ at which $u_{i}$ occurs. $\operatorname{cnt}\left(u_{i}\right)$ is the length of $L\left(u_{i}\right)$. Each entry $l_{k}$, in the postings list $L\left(u_{i}\right)$, has three fields: (1) a sentence identifier, $l_{k}$.sid, (2) the begin position of the particular occurrence of $u_{i}, l_{k}$. first and (3) the end position of the same occurrence of $u_{i}, l_{k}$.last.

We require the input grammar to be the same as that used for named entity annotations in GATE (Cunningham et al., 2002). The GATE architecture for text engineering uses the Java Annotations Pattern Engine (JAPE) (Cunningham, 1999) for its information extraction task. JAPE is a pattern matching language. We support two classes of properties for tokens that are required by grammars such as JAPE: (1) orthographic properties such as an uppercase character followed by lower case characters, and (2) gazetteer (dictionary) containment properties of tokens and token sequences such as 'location' and 'person name'. The set of tokens along with entity types specified by either of these two properties are referred to as Basic Entities. The instances of basic entities specified by orthographic properties must be single tokens. However, instances of basic entities specified using gazetteer containment properties can be token sequences.

The module (1) of our system shown in Figure 1, identifies postings lists for each basic entity type. These postings lists are entered as index entries in $I$ for the corresponding types. For example, if the input rules require tokens/token sequences that satisfy Capsword or Location Dictionary properties, a postings list is created for each of these basic types. Constructing the postings list for a basic entity type with some orthographic property is a fairly straightforward task; the postings lists of tokens satisfying the orthographic properties are merged (while retaining the sorted order of each postings list). The mechanism for generating the postings list of basic entities with gazetteer properties will be developed in the following sections. A rule for NE annotation may require a token to satisfy multiple properties such as Location Dictionary as well as Capsword. The posting list for tokens that satisfy multiple properties are determined by performing an operation parallelint $\left(L, L^{\prime}\right)$ over the posting lists of the corresponding basic entities. The parallelint $\left(L, L^{\prime}\right)$ operation returns a posting list such that each entry in the returned list occurs in both $L$ as well as $L^{\prime}$. The module (2) of our system shown in Figure 1 identifies instances of each annotation type, by performing index-based operations on the postings lists of basic entity types and other tokens.

\section{Annotation using Cascading Regular Expressions}

Regular expressions over basic entities have been extensively used for NE annotations. The Common Pattern Specification Language (CSPL) ${ }^{1}$ specifies a standard for describing Annotators that can be implemented by a series of cascading regular expression matches.

Consider a regular expression $R$ over an alphabet $\Sigma$ of basic entities, and a token sequence

\footnotetext{
${ }^{1}$ http://www.ai.sri.com/ appelt/TextPro
} 


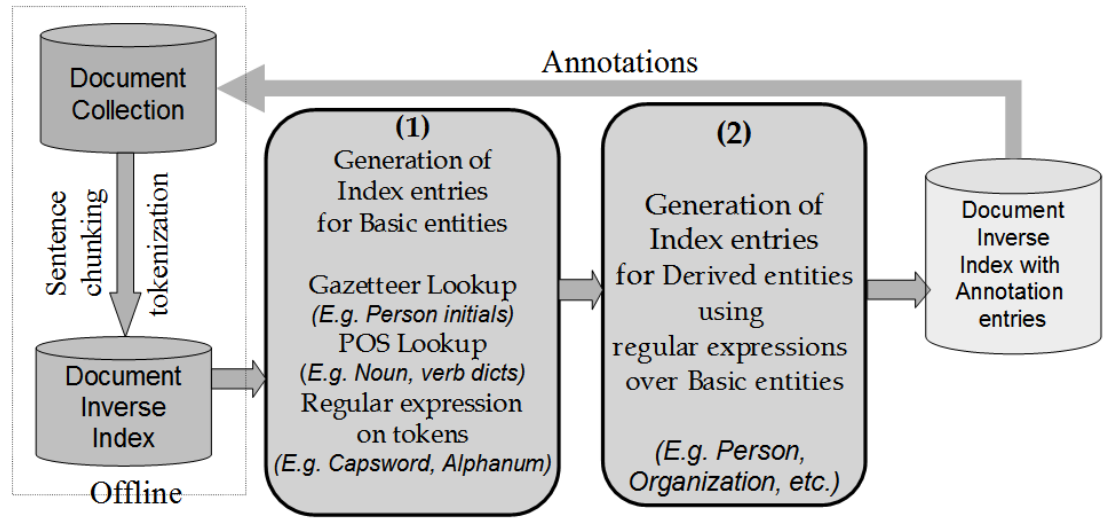

Figure 1: Overview of the entity annotation process described in this paper

$T=\left\{t_{1}, \ldots, t_{W}\right\}$. The annotation problem aims at determining all matches of regular expression $R$ in the token sequence $T$. Additionally, NE annotations do not span multiple sentences. We will therefore assume that the length of any annotated token sequence is bounded by $\Delta$, where $\Delta$ can be the maximum sentence length in the document collection of interest. In practice, $\Delta$ can be even smaller.

\subsection{Computing Annotations using a DFA}

Given a regular expression $R$, we can convert it into a deterministic finite automate (DFA) $D_{R}$. A DFA is a finite state machine, where for each pair of state and input symbol, there is one and only one transition to a next state. $D_{R}$ starts processing of an input sequence from a start state $s_{R}$, and for each input symbol, it makes a transition to a state given by a transition function $\Phi_{R}$. Whenever $D_{R}$ lands in an accept state, the symbol sequence till that point is accepted by $D_{R}$. For simplicity of the document and index algorithms, we will ignore document and sentence boundaries in the following analysis.

Let $@ t_{i, i+\Delta}, 1 \leq i \leq W-\Delta$ be a subsequence of $T$ of length $\Delta$. On a given input $@ t_{i, i+\Delta}, D_{R}$ will determine all token sequences originating at $t_{i}$ that are accepted by the regular expression grammar specified through $D_{R}$. Figure 2 outlines the algorithm findAnnotations that locates all token sequences in $T$ that are accepted by $D_{R}$.

Let $D_{R}$ have $\left\{S_{1}, \ldots, S_{N}\right\}$ states. We assume that the states have been topologically ordered so that $S_{1}$ is the start state. Let $\alpha$ be the time taken to consume a single token and advance the DFA to the next state (this is typically implemented as a table or hash look-up). The time taken by the al-

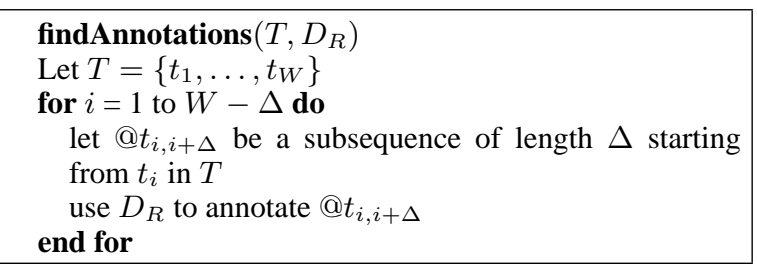

Figure 2: The algorithm for finding all the occurrences of $R$ in a token sequence $T$.

gorithm findAnnotations can be obtained by summing up the number of times each state is visited as the input tokens are consumed. Clearly, the state $S_{1}$ is visited $W$ times, $W$ being the total number of symbols in the token sequence $T$. Let $\operatorname{cnt}\left(S_{i}\right)$ give the total number of times the state $S_{i}$ has been visited. The complexity of this method is:

$$
C_{D}=\alpha \sum_{i=1}^{i=N} \operatorname{cnt}\left(S_{i}\right)=\alpha\left[W+\sum_{i=2}^{i=N} \operatorname{cnt}\left(S_{i}\right)\right]
$$

\subsection{Computing Regular Expression Matches using Index}

In this section, we present a new approach for finding all matches of a regular expression $R$ in a token sequence $T$, based on the inverse index $I$ of $T$. The structure of the inverse index was presented in Section 2. We define two operations on postings lists which find use in our annotation algorithm.

1. merge $\left(L, L^{\prime}\right)$ : Returns a postings list such that each entry in the returned list occurs either in $L$ or $L^{\prime}$ or both. This operation takes $O\left(|L|+\left|L^{\prime}\right|\right)$ time.

2. $\operatorname{consint}\left(L, L^{\prime}\right)$ : Returns a postings list such that each entry in the returned list points to a token sequence which consists of two consecutive 
subsequences @ $s a$ and $@ s b$ within the same sentence, such that, $L$ has an entry for @ $s a$ and $L^{\prime}$ has an entry for @ $s b$. There are several methods for computing this depending on the relative size of $L$ and $L^{\prime}$. If they are roughly equal in size, a simple linear pass through $L$ and $L^{\prime}$, analogous to a merge, can be performed. If there is a significant difference in sizes, a more efficient modified binary search algorithm can be implemented. The details are shown in Figure 3. The

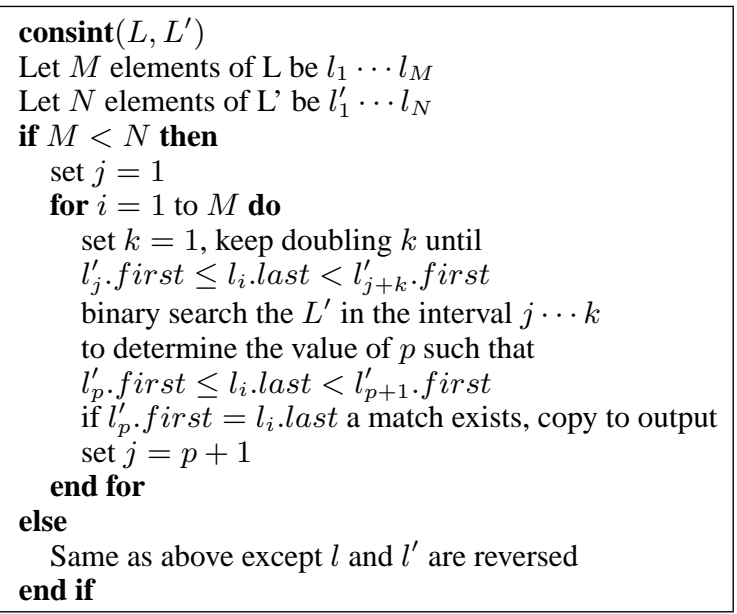

Figure 3: The modified binary search algorithm for consint

complexity of this algorithm is determined by the size $q_{i}$ of the interval required to satisfy $l_{j}^{\prime}$. first $\leq$ $l_{i}$.last $<l_{j+q_{i}}^{\prime}$. first (assuming $|L|<\left|L^{\prime}\right|$ ). It will take an average of $\log _{2}\left(q_{i}\right)$ operations to determine the size of interval and $\log _{2}\left(q_{i}\right)$ operations to perform the binary search, giving a total of $2 \log _{2}\left(q_{i}\right)$. Let $q_{1} \cdots q_{M}$ be the sequence of intervals. Since the intervals will be at most two times larger than the actual interval between the nearest matches in $L^{\prime}$ to $L$, we can see that $\left|L^{\prime}\right| \leq \sum_{i=1}^{M} q_{i} \leq 2 *\left|L^{\prime}\right|$. Hence the worst case will be reached when $q_{i}=2\left|L^{\prime}\right| /|L|$ with a time complexity given by $2|L|\left(\log _{2}\left(\left|L^{\prime}\right| /|L|\right)+1\right)$, assuming $|L|<\left|L^{\prime}\right|$.

To support annotation of a token sequence that matches a regular expression only in the context of some regular expression match on its left and/or right, we implement simple extensions to the consint $\left(L_{1}, L_{2}\right)$ operator. Details of the extensions are left out from this paper owing to space constraints.

\subsection{Implementing a DFA using the Inverse Index}

In this section, we present a method that takes a DFA $D_{R}$ and an inverse index $I$ of a token sequence $T$, to compute a postings list of subsequences of length at most $\Delta$, that match the regular expression $R$.

Let the set $S=\left\{S_{1}, \ldots, S_{N}\right\}$ denote the set of states in $D_{R}$, and let the states be topologically ordered with $S_{1}$ as the start state. We associate an object $l_{i s t_{s, k}}$ with each state $s \in S$ and $\forall 1 \leq k \leq \Delta$. The object list $_{s, k}$ is a posting list of all token sequences of length exactly $k$ that end in state $s$. The list $_{s, k}$ is initialized to be empty for all states and lengths. We iteratively compute list $_{s, k}$ for all the states using the algorithm given in Figure 4. The function $\operatorname{dest}\left(S_{i}\right)$ returns a set of states, such that for each $s \in \operatorname{dest}\left(S_{i}\right)$, there is an arc from state $S_{i}$ to state $s$. The function label $\left(S_{i}, S_{j}\right)$ returns the token associated with the edge $\left(S_{i}, S_{j}\right)$.

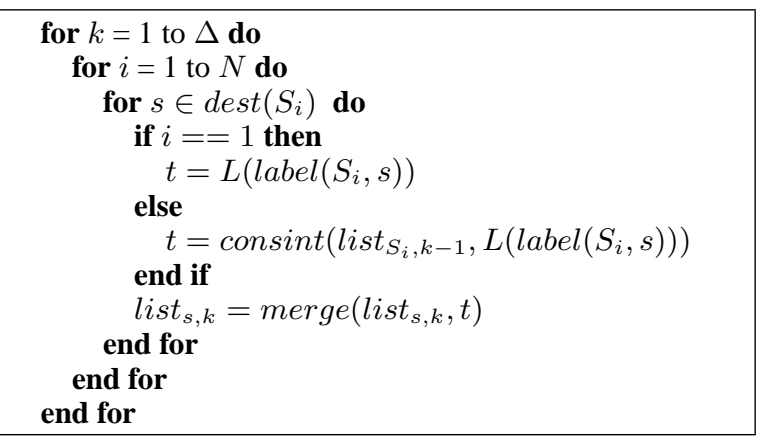

Figure 4: The algorithm for building the index to all token sequences in $T$ that match $R$.

At the end of the algorithm, all token sequences corresponding to postings lists list $_{s, i}, s \in S, 1 \leq$ $i \leq \Delta$ are sequences that are matched by the regular expression $R$.

\subsection{Complexity Analysis for the Index-based Approach}

The complexity analysis of the algorithm given in Figure 4 is based on the observation that, $\sum_{k=1}^{k=\Delta} \mid$ list $_{S_{i}, k} \mid=\operatorname{cnt}\left(S_{i}\right)$. This holds, since list $_{S_{i}, k}$ contains an entry for all sequences that visit the state $S_{i}$ and are of length exactly $k$. Summing the length of these lists for a particular state $S_{i}$ across all the values of $k$ will yield the total number of sequences of length at most $\Delta$ that visit the state $S_{i}$.

For the algorithm in Figure 3, the time taken by 
one consint operation is given by $2 \beta\left(\mid\right.$ list $_{S_{i}, k} \mid *$ $\left.\left(\log \left(\rho_{i j k}\right)+1\right)\right)$ where $\beta$ is a constant that varies with the lower level implementation. $\rho_{i j k}=$ $\frac{\mid L\left(\text { label }\left(S_{i}, S_{j}\right)\right) \mid}{\left|l i s t_{S_{i}, k}\right|}$ is the ratio of the postings list size of the label associated with the arc from $S_{i}$ to $S_{j}$ to the list size of $S_{i}$ at step $k$. Note that $\rho_{i j k} \geq 1$. Let $\operatorname{prev}\left(S_{i}\right)$ be the list of predecessor states to $S_{i}$. The time taken by all the merge operations for a state $S_{i}$ at step $k$ is given by $\gamma\left(\log \left(\left|\operatorname{prev}\left(S_{i}\right)\right|\right) \mid\right.$ list $\left._{S_{i}, k} \mid\right)$ Assuming all the merges are performed simultaneously, $\gamma\left(\log \left(\left|\operatorname{prev}\left(S_{i}\right)\right|\right)\right.$ is the time taken to create each entry in the final merged list, where $\gamma$ is a constant that varies with the lower level implementation. Note this scales as the log of the number of lists that are being merged.

The total time taken by the algorithm given in Figure 4 can be computed using the time spent on merge and consint operations for all states and all lengths. Setting $\overline{\rho_{i s}}=\max _{k} \rho_{i s k}$, the total time $C_{I}$ can be given as:

$C_{I}=\sum_{i=2}^{i=N}\left[\gamma \log \left(\left|\operatorname{prev}\left(S_{i}\right)\right|\right)+2 \beta \sum_{s \in \operatorname{dest}\left(S_{i}\right)} \log \left(\overline{\rho_{i s}}\right)\right] \operatorname{cnt}\left(S_{i}\right)$

Note that in deriving Equation 2, we have ignored the cost of merging $\operatorname{list}\left(S_{a}, k\right)$ for $k=$ $1 \cdots \Delta$ for the accept states.

\subsection{Comparison of Complexities}

To simplify further analysis, we can replace $\operatorname{cnt}\left(S_{i}\right)$ with $f \operatorname{cnt}\left(S_{i}\right)$ where $f \operatorname{cnt}\left(S_{i}\right)=$ $\operatorname{cnt}\left(S_{i}\right) / W$. If we assume that the token distribution statistics of the document collection remain constant as the number of documents increases, we can also assume that $f \operatorname{cnt}\left(S_{i}\right)$ is invariant to $W$. Since $\rho_{i j k}$ is given by a ratio of list sizes, we can also consider it to be invariant to $W$. We now assume $\alpha \approx \beta \approx \gamma$ since these are implementation specific times for similar low level compute operations. With this assumptions from Equations 1 and 2, the ratio $C_{D} / C_{I}$ can be approximated by:

$\frac{1+\sum_{i=2}^{N} f \operatorname{cnt}\left(S_{i}\right)}{\sum_{i=2}^{N}\left[\sum_{s \in \operatorname{dest}\left(S_{i}\right)} 2 \log \left(\overline{\rho_{i s}}\right)+\log \left(\left|\operatorname{prev}\left(S_{i}\right)\right|\right)\right] \operatorname{fcnt}\left(S_{i}\right)}$

The overall ratio of $C_{D}$ to $C_{I}$ is invariant to $W$ and depends on two key factors $f \operatorname{cnt}\left(S_{i}\right)$ and $\sum_{s \in \operatorname{dest}\left(S_{i}\right)} \log \left(\overline{\rho_{\text {is }}}\right)$. If $f \operatorname{cnt}\left(S_{i}\right) \ll 1$, the ratio will be large and the index-based approach will be much faster. However, if either $f \operatorname{cnt}\left(S_{i}\right)$ starts approaching 1 or $\sum_{s \in \operatorname{dest}\left(S_{i}\right)} \log \left(\overline{\rho_{i s}}\right)$ starts getting very large (caused by a large fan out from $S_{i}$ ), the direct match using the DFA may be more efficient.

Intuitively, this makes sense since the main benefit of the index is to eliminate unnecessary hash lookups for tokens do not match the arcs of the DFA. As $f \operatorname{cnt}\left(S_{i}\right)$ approaches 1, this assumption breaks down and hence the inherent efficiency of the direct DFA approach, where only a single hash lookup is required per state regardless of the number of destination states, becomes the dominant factor.

\subsection{Comparison of Complexities for Simple Dictionary DFA}

To illustrate the potential gains from the indexbased annotation, consider a simple DFA $D_{R}$ with two states $S_{1}$ and $S_{2}$. Let the set of unique tokens $A$ be $\{a, b, c \cdots z\}$. Let $E$ be the dictionary $\{a, e, i, o, u\}$. Let $D_{R}$ have five arcs from $S_{1}$ to $S_{2}$ one for each element in $E$. The DFA $D_{R}$ is a simple acceptor for the dictionary $E$, and if run over a token sequence $T$ drawn from $A$, it will match any single token that is in $E$. For this simple case $f \operatorname{cnt}\left(S_{2}\right)$ is just the fraction of tokens that occur in $E$ and hence by definition $f \operatorname{cnt}\left(S_{2}\right) \leq 1$. Substituting into 3 we get

$$
\frac{C_{D}}{C_{I}}=\frac{1+f \operatorname{cnt}\left(S_{2}\right)}{2 \log (5) f \operatorname{cnt}\left(S_{2}\right)}
$$

As long as $f \operatorname{cnt}\left(S_{2}\right)<0.27$, this ratio will always be greater than 1 .

\section{Inverse Index-based Annotation using Regular Expressions}

A DFA corresponding to a given regular expression can be used for annotation, using the inverse index approach as described in Section 3.3. However, the NFA to DFA conversion step may result in a DFA with a very large number of states. We develop an alternative algorithm that translates the original regular expression directly into an ordered AND/OR graph. Associated with each node in the graph is a regular expression and a postings list that points to all the matches for the node's regular expression in the document collection. There are two node types: AND nodes where the output list is computed from the consint of the postings lists of two children nodes and OR nodes where the output list is formed by merging the posting 
lists of all the children nodes. Additionally, each node has two binary properties: isOpt and selfLoop. The first property is set if the regular expression being matched is of the form ' $R$ ?', where '?' denotes that the regular expression $\mathrm{R}$ is optional. The second property is set if the regular expression is of the form ' $\mathrm{R}+$ ', where ' + ' is the Kleen operator denoting one or more occurrences. For the case of ' $\mathrm{R}$ *', both properties are set.

The AND/OR graph is recursively built by scanning the regular expression from left to right and identifying every sub-regular expression for which a sub-graph can be built. We use capital letters $R, X$ to denote regular expressions and small letters $a, b, c$, etc., to denote terminal symbols in the symbol set $\Sigma$. Figure 5 details the algorithm used to build the AND/OR graph. Effectively, the AND/OR graph decomposes the computation of the postings list for $R$ into a ordered set of merge and consint operations, such that the output $L(v)$ for node $v$ become the input to its parents. The graph specifies the ordering, and by evaluating all the nodes in dependency order, the root node will end up with a postings list that corresponds to the desired regular expression.

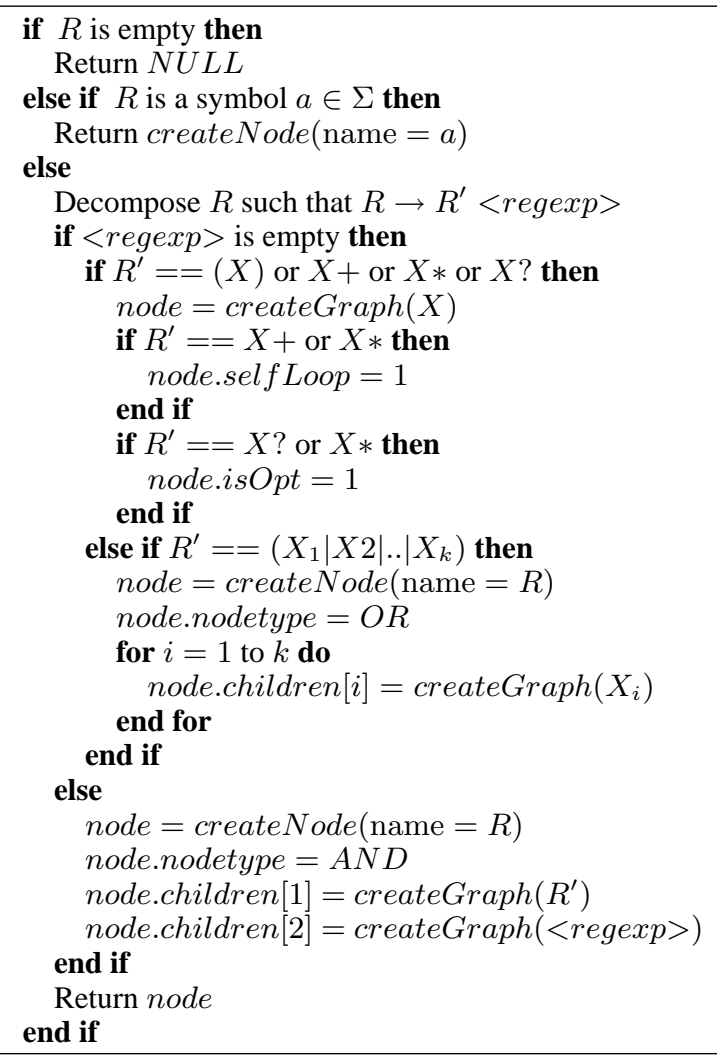

Figure 5: createGraph $(R)$

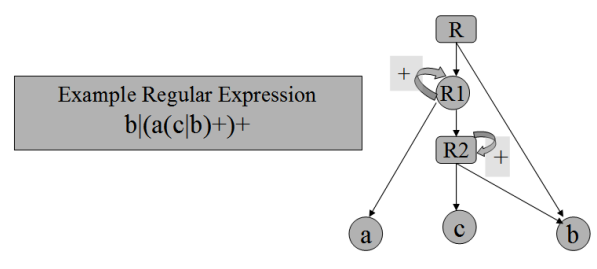

Figure 6: An example regular expression and corresponding AND/OR graph

\subsection{Handling '?' and Kleen Operators}

The isOpt and selfLoop properties of a node are set if the corresponding regular expression is of the form $R$ ?, $R+$ or $R *$. To handle the $R$ ? case we associate a new property is $O p t$ with the output list $L(v)$ from node $v$, such that $L(v) . i s O p t=1$ if the $v . i s O p t=1$. We also define two operations consint $_{\epsilon}$ in Figure 7 and merge $\epsilon_{\epsilon}$ which account for the isOpt property of their argument lists. For consint $_{\epsilon}$, the generated list has its $i s O p t$ set to 1 if and only if both the argument lists have their

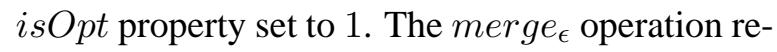
mains the same as merge, except that the resultant list has $i s O p t$ set to 1 if any of its argument lists has $i s O p t$ set to 1 . The worst case time taken by consint $_{\epsilon}$ is bounded by 1 consint and 2 merge operations.

To handle the $R+$ case, we define a new operator $\operatorname{consint}_{\epsilon}(L,+)$ which returns a postings list $L^{\prime}$, such that each entry in the returned list points to a token sequence consisting of all $k \in[1, \Delta]$ consecutive subsequences @ $s_{1}$, $s_{2} \ldots$ @ $s_{k}$, each @ $s_{i}, 1 \leq i \leq k$ being an entry in $L$. A simple linear pass through $L$ is sufficient to obtain consint $(L,+)$. The time complexity of this operation is linear in the size of $L^{\prime}$. The isOpt property of the result list $L^{\prime}$ is set to the same value as its argument list $L$.

Figure 6 shows an example regular expression and its corresponding AND/OR graph; AND nodes are shown as circles whereas OR nodes are shown as square boxes. Nodes having isOpt and selfLoop properties are labeled with,$+ *$ or ?. Any AND/OR graph thus constructed is acyclic. The edges in the graph represent dependency between computing nodes. The main regular expression is at the root node of the graph. The leaf nodes correspond to symbols in $\Sigma$. Figure 8 outlines the algorithm for computing the postings list of a regular expression by operating bottom-up on the AND/OR graph. 


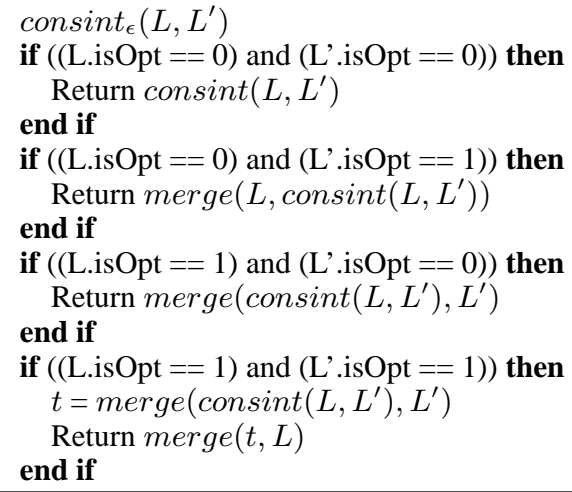

Figure 7: consint $_{\epsilon}$

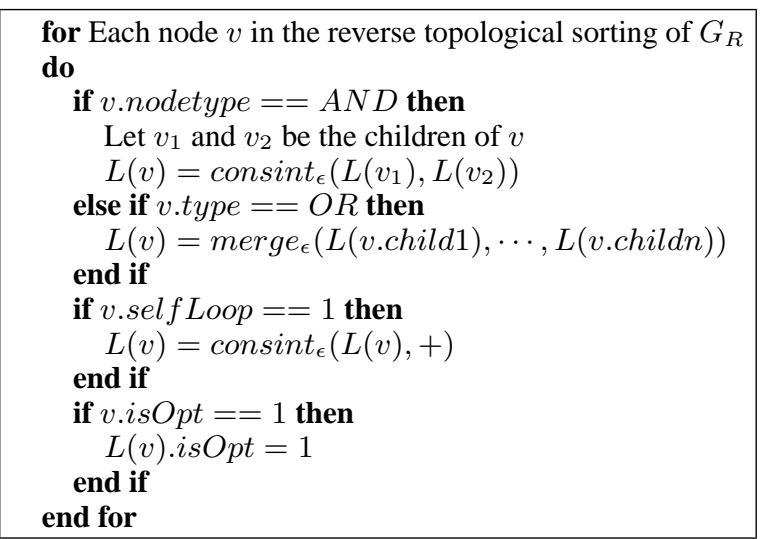

Figure 8: The algorithm for computing postings list of a regular expression $R$ using the inverse index $I$ and the corresponding AND/OR graph $G_{R}$

\section{Experiments and Results}

In this section, we present empirical comparison of performance of the index-based annotation technique (Section 4) against annotation based on the 'document paradigm' using GATE. The experiments were performed on two data sets, viz., (i) the enron email data $\operatorname{set}^{2}$ and (ii) a combination of Reuters-21578 data set ${ }^{3}$ and the 20 Newsgroups data set ${ }^{4}$. After cleaning, the former data set was $2.3 \mathrm{~GB}$ while the latter was $93 \mathrm{MB}$ in size. Our code is entirely in Java. The experiments were performed on a dual $3.2 \mathrm{GHz}$ Xeon server with 4 GB RAM. The code for creation of the index was custom-built in Java. Prior to indexing, the sentence segmentation and tokenization of each data set was performed using in-house Java versions of

\footnotetext{
${ }^{2}$ http://www. cs. cmu.edu/ enron/

${ }^{3}$ http: //www.daviddlewis.com/resources / testcollections/reuters21578/

${ }^{4}$ http://people.csail.mit.edu/jrennie/ 20Newsgroups /
}

standard tools 5 .

\subsection{Rule Specification using JAPE}

JAPE is a version of CPSL $^{6}$ (Common Pattern Specification Language). JAPE provides finite state transduction over annotations based on regular expressions. The JAPE grammar requires information from two main resources: (i) a tokenizer and (ii) a gazetteer.

(1) Tokenizer: The tokenizer splits the text into very simple tokens such as numbers, punctuation and words of different types. For example, one might distinguish between words in uppercase and lowercase, and between certain types of punctuation. Although the tokenizer is ca pable of much deeper analysis than this, the aim is to limit its work to maximise efficiency, and enable greater flexibility by placing the burden on the grammar rules, which are more adaptable. A rule has a left hand side (LHS) and a right hand side (RHS). The LHS is a regular expression which has to be matched on the input; the RHS describes the annotations to be added to the Annotation Set. The LHS is separated from the RHS by ' $>$ '. The following four operators can be used on the LHS: '|', '?', '*' and '+'. The RHS uses ';' as a separator between statements that set the values of the different attributes. The following tokenizer rule identifies each character sequence that begins with a letter in upper case and is followed by 0 or more letters in lower case:

"UPPERCASELETTER" "LOWERCASELETTER" * >> Token; orth=upperInitial; kind=word;

Each such character sequence will be annotated as type "Token". The attribute "orth" (orthography) has the value "upperInitial"; the attribute "kind" has the value "word".

(2) Gazetteer: The gazetteer lists used are plain text files, with one entry per line. Each list represents a set of names, such as names of cities, organizations, days of the week, etc. An index file is used to access these lists; for each list, a major type is specified and, optionally, a minor type. These lists are compiled into finite state machines. Any text tokens that are matched by these machines will be annotated with features specifying the major and minor types. JAPE grammar rules

\footnotetext{
${ }^{5}$ http://12r.cs.uiuc.edu/ $\sim_{\text {Cogcomp / }}$ tools.php

${ }^{6} \mathrm{~A}$ good description of the original version of this language is in Doug Appelt's TextPro manual: http://www . ai.sri.com/ appelt/TextPro.
} 
then specify the types to be identified in particular circumstances.

The JAPE Rule: Each JAPE rule has two parts, separated by "->". The LHS consists of an annotation pattern to be matched; the RHS describes the annotation to be assigned. A basic rule is given as:

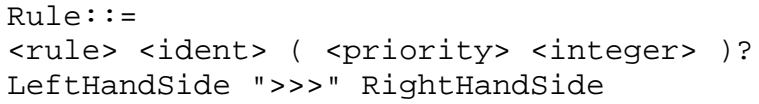

(1) Left hand side: On the LHS, the pattern is described in terms of the annotations already assigned by the tokenizer and gazetteer. The annotation pattern may contain regular expression operators $($ e.g. $*, ?,+)$. There are 3 main ways in which the pattern can be specified:

1. value: specify a string of text, e.g. \{Token.string $==$ "of" $\}$

2. attribute: specify the attributes (and values) of a token (or any other annotation), e.g. $\{$ Token.kind $==$ number $\}$

3. annotation: specify an annotation type from the gazetteer, e.g. $\quad$ Lookup.minorType $==$ month\}

(2) Right hand side: The RHS consists of details of the annotations and optional features to be created. Annotations matched on the LHS of a rule may be referred to on the RHS by means of labels that are attached to pattern elements. Finally, attributes and their corresponding values are added to the annotation. An example of a complete rule is:

Rule: NumbersAndUnit

( $(\{$ Token $\cdot$ kind $==$ "number" $\})+$ : numbers

$\{$ Token.kind=="unit" $\}$ )

$>>>$

: numbers. Name $=\{$ rule $=$ "NumbersAndUnit $"\}$

This says 'match sequences of numbers followed by a unit; create a Name annotation across the span of the numbers, and attribute rule with value NumbersAndUnit'.

Use of context: Context can be dealt with in the grammar rules in the following way. The pattern to be annotated is always enclosed by a set of round brackets. If preceding context is to be included in the rule, this is placed before this set of brackets. This context is described in exactly the same way as the pattern to be matched. If context following the pattern needs to be included, it is placed

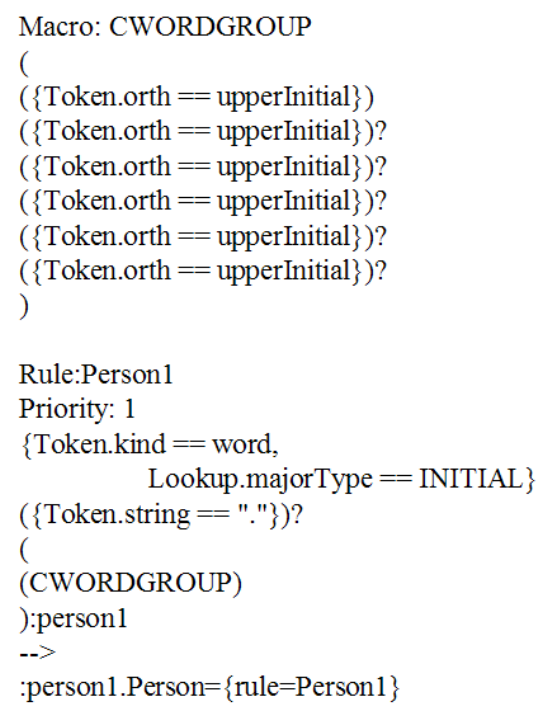

Figure 9: An example JAPE rule used in the experiments

after the label given to the annotation. Context is used where a pattern should only be recognised if it occurs in a certain situation, but the context itself does not form part of the pattern to be annotated.

For example, the following rule for 'email-id's (assuming an appropriate regular expression for "EMAIL-ADD") would mean that an email address would only be recognized if it occurred inside angled brackets (which would not themselves form part of the entity):

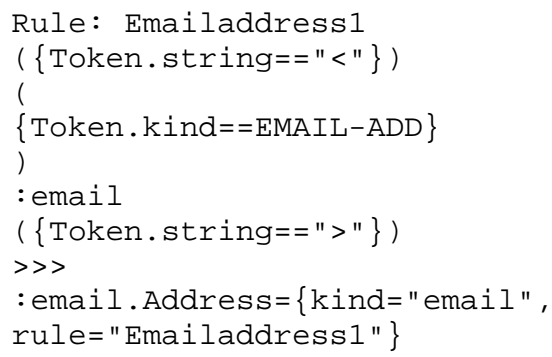

\subsection{Results}

In our first experiment, we performed annotation of the two corpora for 4 annotation types using 2 JAPE rules for each type. The 4 annotation types were 'Person name', 'Organization', 'Location' and 'Date'. A sample JAPE rule for identifying person names is shown in Figure 9. This rule identifies a sequence of words as a person name when each word in the sequence starts with an alphabet in upper-case and when the sequence is immediately preceded by a word from a dictionary of 'INITIAL's. Example words in the 'INITIAL' dictionary are: 'Mr.', 'Dr.', 'Lt.', etc. 
Table 1 compares the time taken by the indexbased annotator against that taken by GATE for the $8 \mathrm{JAPE}$ rules. The index-based annotator performs 8-13 times faster than GATE. Table 2 splits the time mentioned for the index-based annotator in Table 1 into the time taken for the task of computing postings lists for basic entities and derived entities (c.f. Section 2) for each of the data sets. We can also observe that a greater speedup is achieved for the larger corpus.

\begin{tabular}{|c|r|r|}
\hline Data set & GATE & Index-based \\
\hline Enron & 4974343 & 374926 \\
\hline Reuters & 752287 & 92238 \\
\hline
\end{tabular}

Table 1: Time (in milliseconds) for computing annotations using the two techniques

\begin{tabular}{|c|r|r|r|}
\hline Data set & $\begin{array}{r}\text { Orthographic } \\
\text { entity types }\end{array}$ & $\begin{array}{r}\text { Gazetteer } \\
\text { entity types }\end{array}$ & $\begin{array}{r}\text { Derived } \\
\text { entity types }\end{array}$ \\
\hline Enron & 38285 & 105870 & 230771 \\
\hline Reuters & 28493 & 21531 & 42214 \\
\hline
\end{tabular}

Table 2: Time (in milliseconds) for computing postings lists of entity types

An important advantage of performing annotations over the inverse index is that index entries for basic entity types can be preserved and reused for annotation types as additional rules for annotation are specified by users. For instance, the index entry for 'Capsword' might find reuse in several annotation rules. As against this, a documentbased annotator has to process each document from scratch for every newly introduced annotation rule. To verify this, we introduced 1 additional rule for each of the 4 named entity types. In Table 3, we compare the time required by the index-based annotator against that required by GATE for annotating the two corpora using the 4 additional rules. We achieve a greater speedup factor of 23-37 for incremental annotation.

\begin{tabular}{|c|r|r|}
\hline Data set & GATE & Index-based \\
\hline Enron & 1479954 & 62227 \\
\hline Reuters & 661157 & 17929 \\
\hline
\end{tabular}

Table 3: Time (in milliseconds) for computing annotations using the two techniques for the additional 4 rules

\section{Conclusions}

In this paper we demonstrated that a suitably constructed inverse index contains all the necessary information to implement entity annotators that use cascading regular expressions. The approach has the key advantage of not requiring access to the original unstructured data to compute the annotations. The method uses a basic set of operators on the inverse index to construct indexes to all matches for a regular expression in the tokenized data set. We showed theoretically, that for a DFA implementation, the index approach can be much faster if the index sizes corresponding to the labels on the DFA are a small fraction of the total number of tokens in the data set. We also provided a more efficient index-based implementation that is directly computed from the regular expressions without the need of a DFA conversion and experimentally demonstrated the gains.

\section{References}

Eugene Agichtein and Luis Gravano. 2000. Snowball: Extracting relations from large plain-text collections. In Proceedings of the Fifth ACM International Conference on Digital Libraries.

Junghoo Cho and Sridhar Rajagopalan. 2002. A fast regular expression indexing engine. In Proceedings of the $18^{\text {th }}$ International Conference on Data Engineering.

Brian Cooper, Neal Sample, Michael J. Franklin, Gísli R. Hjaltason, and Moshe Shadmon. 2001. A fast index for semistructured data. In The VLDB Conference, pages 341-350.

H. Cunningham, D. Maynard, K. Bontcheva, and V. Tablan. 2002. GATE: A framework and graphical development environment for robust NLP tools and applications.

H. Cunningham. 1999. Jape - a java annotation patterns engine.

Line Eikvil. 1999. Information extraction from world wide web - a survey. Technical Report 945, Norweigan Computing Center.

Quanzhong Li and Bongki Moon. 2001. Indexing and querying XML data for regular path expressions. In The VLDB Journal, pages 361-370.

Andrew McCallum, Dayne Freitag, and Fernando Pereira. 2000. Maximum entropy Markov models for information extraction and segmentation. In Proc. 17th International Conf. on Machine Learning, pages 591-598. Morgan Kaufmann, San Francisco, CA. 